\title{
Implications of non-sub-wavelength resonator spacing on the sound transmission loss predictions of locally resonant metamaterial partitions
}

\author{
Lucas Van Belle* \\ DMMS lab, Flanders Make \\ Division LMSD, Department of Mechanical Engineering \\ $\mathrm{KU}$ Leuven \\ Celestijnenlaan 300 - box 2420 \\ Heverlee B-3001, Belgium \\ e-mail: lucas.vanbelle@kuleuven.be \\ Claus Claeys \\ DMMS lab, Flanders Make \\ Division LMSD, Department of Mechanical Engineering \\ $\mathrm{KU}$ Leuven \\ Celestijnenlaan 300 - box 2420 \\ Heverlee B-3001, Belgium \\ e-mail: claus.claeys@kuleuven.be \\ Elke Deckers \\ DMMS lab, Flanders Make \\ Division LMSD, Department of Mechanical Engineering \\ KU Leuven, Campus Diepenbeek \\ Wetenschapspark 27 \\ Diepenbeek B-3590, Belgium \\ e-mail: elke.deckers@kuleuven.be \\ Wim Desmet \\ DMMS lab, Flanders Make \\ Division LMSD, Department of Mechanical Engineering \\ KU Leuven \\ Celestijnenlaan 300 - box 2420 \\ Heverlee B-3001, Belgium \\ e-mail:wim.desmet@kuleuven.be
}

Locally resonant metamaterials have recently emerged and gained attention in the field of noise control engineering. The addition of resonant structures to a flexible partition on a sub-wavelength scale enables a targeted frequency range of strongly reduced vibration and sound transmission. These structures have been widely studied and are typically analyzed using infinite periodic structure theory. The implications of non-sub-wavelength resonator spacing on the sound transmission loss of metamaterial partitions as well as on the representativeness of the infinite periodic structure modeling are, however, less well known. In this technical brief it is shown that, although a shifted sound transmission loss peak can be predicted for partitions with non-sub-wavelength resonator spacing when using infinite periodic structure modeling, the sound transmission loss enhancement is not guaranteed for their finite structure counterparts. 


\section{Introduction}

In the past decades, locally resonant metamaterials have come to the fore as potential lightweight solutions with favorable noise and vibration attenuation performance in targeted frequency ranges. By adding or embedding resonant structures to or in a flexible host structure on a sub-wavelength scale, a resonance-based stop band for elastic wave propagation can be obtained around the tuned resonator frequency, enabling strong vibration reduction $[1,2]$. A resonance-based stop band for the bending waves in partitions can create a targeted frequency range of strongly increased sound transmission loss (STL) around the tuned resonator frequency, outperforming the acoustic mass-law $[1,3]$. Such metamaterial partitions have been widely studied in recent years and their potential has been experimentally demonstrated in, amongst others, single panels $[4,5]$, double panels $[6,7]$, sandwich panels [8] and enclosures [9].

The vibro-acoustic performance of these often periodic structures is typically analyzed using infinite periodic structure modeling. Their stop bands are generally predicted using dispersion curves for structural wave propagation based on a unit cell model $[2,10]$. To predict the STL, a variety of infinite periodic structure models is commonly applied. Finite element based unit cell modeling $[4,11,12]$ models the structure in detail, but can be computationally expensive. Therefore, simplified models such as effective medium models $[3,13,14]$ or analytical models [15-17] are often preferred. These are more affordable, but sometimes limited to the (deep) sub-wavelength regime, as they simplify or homogenize the complex dynamic behavior. In some cases, e.g. [18], the problem is even reduced to a two degreesof-freedom system, discarding host structure dynamics and vibro-acoustic interactions. Care should hence be taken in the application of these models, in particular when leaving the (deep) sub-wavelength regime.

Although near-sub-wavelength tuning has been proposed as a means to broaden the vibration attenuation frequency range due to the combination between resonancebased and Bragg-interference-based stop bands [19], a nonsub-wavelength resonator spacing is known to no longer guarantee resonance-based stop bands [20]. The implications of non-sub-wavelength resonator spacing on the STL of metamaterial partitions are, however, less well known. In several studies, non-sub-wavelength spaced resonators have been considered [13, 16,21] without particular consideration of their non-sub-wavelength nature or of the validity of the applied infinite periodic structure models. The latter are not guaranteed to be valid outside their intended (deep) subwavelength regime and can hence obscure effects of the nonsub-wavelength resonator spacing. Moreover, contrary to the often reported STL peak occurring precisely at the tuned resonance frequency of the resonators using simplified models, Xiao et al. [22] recently explicitly pointed out that, with increasing resonator spacing, the STL peak frequency of an infinite periodic metamaterial partition always decreases with respect to the tuned resonator frequency. This effect of the hybridization between the resonator mode and the waves in the host structure was also found for non-sub-wavelength resonator spacing, but no verification of these observations was carried out to investigate the validity of these observations for finite structures.

Identifying potential differences between infinite and finite structure performance predictions is in particular important. For (deep) sub-wavelength resonator spacing, infinite periodic structure modeling can suffice to describe the finite structure performance: the non-resonant sound transmission can be captured by the altered dynamic mass density of the partition and the STL peak around the tuned resonator frequency corresponds to a strong increase of the dynamic mass density $[3,7]$. For non-sub-wavelength resonator spacing, however, the absence of a stop band and a shift of the STL peak frequency could enable a strong influence of the finite partition resonances on the STL performance, which is not accounted for in infinite structure modeling. While non-sub-wavelength resonator spacing is explicitly considered in finite metamaterial plates in [20], only vibration attenuation is considered and related to stop band behavior. Also in [22] non-sub-wavelength resonator spacing is explicitly investigated, but only infinite periodic structure STL predictions are performed. Hence, to account for the influence of finite structure effects on the STL performance of metamaterial partitions of realizable dimensions, finite structure dimensions should be accounted for $[4,17,23]$. Aside from experimental validations of metamaterial partitions with subwavelength resonator spacing, however, the STL analysis of finite-sized metamaterial partitions of representative dimensions, in particular for non-sub-wavelength resonator spacing, is limited in current literature.

To gain a better understanding of the implications of non-sub-wavelength resonator spacing on the vibro-acoustic performance of metamaterial partitions and of potential gaps arising from the use of infinite periodic structure models, this technical brief further analyzes the effect of relaxing the sub-wavelength resonator tuning on the stop band, vibration attenuation and STL of plates with periodically added resonators. To this end, finite element based infinite periodic and finite structure models are used, which are not limited to the sub-wavelength regime. The rest of this paper is organized as follows. After describing the problem setting in Sec. 2, the modeling methods are explained in Sec. 3. Next, the results are presented and discussed in Sec. 4 and the conclusions are summarized in Sec. 5.

\section{Problem description}

The stop band behavior, vibration response and STL of a $1 \mathrm{~mm}$ thick aluminum plate (Tab. 1) with periodically added mass-spring resonators is analysed (Fig. 1a). The resonators are added with spatial period $L_{x} \times L_{y}=0.05 \times 0.05 \mathrm{~m}$ in the $x y$-plane and target the acoustically relevant out-of-plane bending wave vibrations. This periodic structure can be represented by a single $L_{x} \times L_{y}$ sized unit cell (Fig. 1b).

The resonators add $20 \%$ of mass to the plate host structure. Before choosing the resonator tuning, the frequency ratio $f_{\text {ratio }}$ is introduced to be able to describe the sub- 


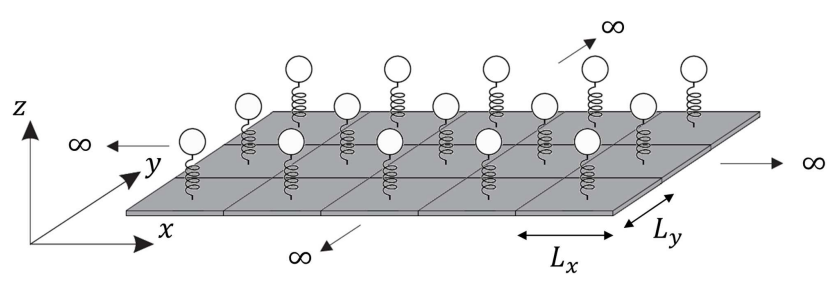

(a)

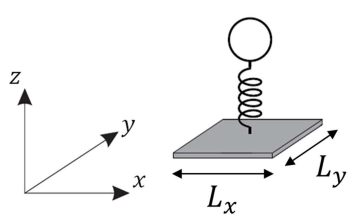

(b)

Fig. 1: Plate with periodically added mass-spring resonators (a) and its representative unit cell (b).

Young's modulus $E$ mass density $\rho$ Poisson's ratio $v$

$70 \mathrm{GPa} \quad 2700 \mathrm{~kg} / \mathrm{m}^{3} \quad 0.3$

Table 1: Properties of the aluminum plate host structure.

\begin{tabular}{l|rrrrr}
$f_{\text {res }}[\mathrm{Hz}]$ & 500 & 1000 & 1500 & 2000 & 2500 \\
\hline$f_{\text {ratio }}$ & 0.52 & 1.03 & 1.55 & 2.07 & 2.58
\end{tabular}

Table 2: Tuned natural frequencies $f_{\text {res }}$ of the resonators and corresponding frequency ratios $f_{\text {ratio }}$.

wavelength requirement, following [20]:

$$
f_{\text {ratio }}=\frac{f_{\text {res }}}{f_{\lambda / 2}},
$$

with $f_{\text {res }}$ the tuned natural frequency of the resonator and $f_{\lambda / 2}$ the Bragg interference limit at which half the wavelength $\lambda$ of the targeted wave type in the host structure corresponds to the periodic spacing between adjacent resonators. For bending waves in a thin plate with thickness $h, f_{\lambda / 2}$ of a square unit cell with $L_{U C}=L_{x}=L_{y}$ is [20]:

$$
f_{\lambda / 2}=\frac{\pi}{2 L_{U C}^{2}} \sqrt{\frac{E h^{2}}{12 \rho\left(1-v^{2}\right)}} .
$$

For the considered plate and unit cell size $f_{\lambda / 2}=968 \mathrm{~Hz}$. To satisfy the sub-wavelength criterion for guaranteed resonance-based stop band behavior around $f_{\text {res }}$, an $f_{\text {ratio }}<1$ is required [20]. To analyze the impact of relaxing this criterion, $f_{\text {res }}$ is varied from $500 \mathrm{~Hz}$ to $2500 \mathrm{~Hz}$, leading to an $f_{\text {ratio }}$ between 0.52 and 2.58 (Tab. 2).

For ambient air with soundspeed $c_{a}=340 \mathrm{~m} / \mathrm{s}$ and mass density $\rho_{a}=1.225 \mathrm{~kg} / \mathrm{m}^{3}$, the critical vibro-acoustic coincidence frequency of the plate host structure is $11940 \mathrm{~Hz}$. At this frequency, the bending wavenumber in the partition is equal to the acoustic wavenumber in the air and the plate becomes an efficient sound radiator, strongly reducing the STL [24]. Since this coincidence frequency lies well above the considered $f_{\text {res }}$, it will hence not influence this study $[3,25]$.

\section{Methodology}

This section gives an overview of the applied methodologies for the calculation of dispersion curves, finite structure vibration response and infinite and finite structure STL.

\subsection{Dispersion curves}

To analyze the bending wave stop band behavior of the periodic plates, dispersion curves for free structural wave propagation are calculated, considering an infinite periodic structure. To this end, the wave finite element method is applied [10]. The undamped unit cell of the structure is discretized using finite elements in COMSOL MULTIPHYSICS: $10 \times 10$ shell elements for the host structure and a lumped point mass and spring element for the resonator, with stiffness normal to the plate. Bloch-Floquet periodicity boundary conditions are imposed to the unit cell boundaries. By prescribing real wave propagation constants $\left(\mu_{x}, \mu_{y}\right)$ along the unit cell's irreducible Brillouin contour (IBC) $\operatorname{Re}\left(\mu_{x}, \mu_{y}\right)$ : $\Gamma, \mathrm{X}, \mathrm{M}, \Gamma \mapsto(0,0),(\pi, 0),(\pi, \pi),(0,0)$, the resulting dispersion eigenvalue problem is solved to real frequencies $[2,10]$.

\subsection{Vibration response}

The out-of-plane vibration response of a simply supported $60 \times 45 \mathrm{~cm}$ finite plate, consisting of $12 \times 9$ unit cells, is analyzed. The finite plate is excited by a slightly off-center time-harmonic unit point force $F=1 \mathrm{~N}$ in the normal direction at $(0.295,0.220) \mathrm{m}$. The vibration response is calculated using SiEMENS LMS VIRTUAL.LAB by applying the classical finite element method [26]. The model, constructed in MSC PATRAN, comprises 10800 shell elements for the host structure and 108 lumped point mass and spring elements for the resonators. The global vibration response is assessed by means of the root mean square (RMS) average time-harmonic out-of-plane displacement of all the plate nodes. To assess the local vibration response at the resonator bases, the RMS average out-of-plane displacement of the plate nodes to which the resonators are attached is evaluated.

\subsection{Sound transmission loss}

For the STL prediction, both full infinite periodic structure and finite structure modeling are applied, as in [4]. A normally incident acoustic plane wave excitation is considered, which is often used to assess the STL of metamaterial panels, and which will already allow to reveal significant differences between infinite and finite structure STL predictions in Sec. 4.3.

The STL of the infinite periodic plate is calculated using the hybrid wave based - finite element method [27]. To this end, the finite element unit cell of Sec. 3.1 is strongly cou- 
pled to two semi-unbounded periodic acoustic wave based domains, above and below the structure, forming a vibroacoustic unit cell. The STL of a simply supported $60 \times 45 \mathrm{~cm}$ finite plate, consisting of $12 \times 9$ unit cells, is analyzed using SiEMENS LMS VIRTUAL.LAB by means of a classical vibro-acoustic finite element model. The finite structure model of Sec. 3.2 is strongly coupled to two unbounded acoustic finite element half-spaces, above and below the structure, leading to a total of 21600 acoustic elements. Automatically matched layer (AML) boundary conditions are applied to the truncated acoustic domain boundaries to model the free field radiation $[4,28,29]$.

\section{Results}

In this section, the implications of non-sub-wavelength resonator spacing on the stop band, finite structure vibration response and infinite and finite structure STL are analyzed.

\subsection{Dispersion curves}

Dispersion curves are calculated along the IBC in the frequency range up to $3000 \mathrm{~Hz}$ (Fig. 2). For $f_{\text {res }}=500 \mathrm{~Hz}$, the sub-wavelength criterion is satisfied and a resonancebased stop band is obtained for the bending waves between $485-542 \mathrm{~Hz}$. For $f_{\text {res }}=1000 \mathrm{~Hz}$ a transition takes place: the sub-wavelength criterion is no longer satisfied, but a stop band is still obtained between $878-967 \mathrm{~Hz}$, below $f_{\text {res. }}$. However, the nature of the stop band changes from a resonance-based stop band to a stop band with Bragg interference characteristics, no longer including $f_{\text {res }}$ and limiting the upper stop band frequency of this transition stop band to $f_{\lambda / 2}[19,20]$. By further increasing $f_{r e s}>f_{\lambda / 2}$, the resonance-based stop band is no longer guaranteed [20], while no interference-based stop band emerges around $f_{\lambda / 2}$ for the considered $m_{\text {ratio }}$. Consequently, for $f_{\text {res }}=1500 \mathrm{~Hz}$, $2000 \mathrm{~Hz}$ and $2500 \mathrm{~Hz}$, no bending wave stop band is obtained. Corresponding to the findings of Xiao et al. [30], directional band gaps can, however, still be observed above $f_{\lambda / 2}$ near $f_{\text {res }}$ for the considered $f_{\text {res }}>f_{\lambda / 2}$, as is the case along e.g. ГX (Fig. 2b-2e).

\subsection{Vibration response}

The out-of-plane vibration response of a finite plate is calculated for frequencies $f=1-1-3000 \mathrm{~Hz}$ to verify the influence of relaxing the sub-wavelength requirement for realizable structure dimensions on the vibration attenuation (Fig. 3). The resonance-based stop band for $f_{\text {res }}=500 \mathrm{~Hz}$ results in a distinct zone of strong vibration attenuation and without bending modes for the finite plate. For $f_{\text {res }}=$ $1000 \mathrm{~Hz}$, less strong vibration attenuation is obtained in the transition stop band. The stop band disappears when $f_{\text {res }}$ is further increased to $1500 \mathrm{~Hz}$ and higher, which results in the absence of a distinct zone of vibration attenuation without structural modes. The modal density in the frequency range around $f_{\text {res }}$ is, however, slightly reduced for $f_{\text {res }}=1500 \mathrm{~Hz}$ and $f_{\text {res }}=2000 \mathrm{~Hz}$. This may originate from the directional band gaps around these $f_{\text {res }}$, as observed before in the dispersion curves, partly inhibiting the build-up of structural modes along the corresponding propagation directions.

The vibration response is analyzed in more detail by inspecting the response of the resonator bases (Fig. 4). The resonators clearly lead to local vibration suppression at $f_{\text {res }}$, which results in global vibration reduction in the stop band frequency range around $f_{\text {res }}$ when the sub-wavelength criterion is satisfied [20]. When $f_{\text {ratio }} \approx 1$, the global vibration reduction in the stop band originates from both interference and local resonance effects, as the strongest vibration reduction in the plate at $885 \mathrm{~Hz}$ is shifted considerably down in frequency with respect to $f_{\text {res }}$. For higher $f_{\text {ratio }}>1$, the stop band has disappeared. Despite the local vibration suppression at the resonator bases, no distinct global vibration reduction is achieved. As shown in [20], this results from the existence of freely propagating waves, for which the resonators are located in nodal positions. A non-sub-wavelength resonator spacing does, hence, not guarantee global vibration reduction.

\subsection{Sound transmission loss}

The main interest lies in the influence of relaxing the sub-wavelength criterion on the STL and in verifying if the observations for infinite periodic structures are retrieved for their finite counterparts. First, the infinite periodic structure STL is calculated for frequencies up to $3000 \mathrm{~Hz}$ (Fig. 5).

An STL peak is obtained for all $f_{\text {res }}$, followed by an STL dip, regardless off the sub-wavelength criterion and the presence of a resonance-based stop band. The STL peak and dip frequencies $f_{\text {peak }}$ and $f_{\text {dip }}$ are listed in Tab. 3.

\begin{tabular}{ccccc}
$f_{\text {res }}[\mathrm{Hz}]$ & $f_{\text {ratio }}$ & stop band $[\mathrm{Hz}]$ & $f_{\text {peak }}[\mathrm{Hz}]$ & $f_{\text {dip }}[\mathrm{Hz}]$ \\
\hline 500 & 0.52 & $485-542$ & 495 & 542 \\
1000 & 1.03 & $878-967$ & 960 & 1051 \\
1500 & 1.55 & $/$ & 1368 & 1496 \\
2000 & 2.07 & $/$ & 1701 & 1854 \\
2500 & 2.58 & $/$ & 1955 & 2122 \\
\hline
\end{tabular}

Table 3: Tuned resonator frequencies, frequency ratios, predicted bending wave stop bands, and STL peak and dip frequencies $f_{\text {peak }}$ and $f_{\text {dip }}$ for the infinite plates with periodically added resonators.

For the sub-wavelength tuning $f_{\text {res }}=500 \mathrm{~Hz}$, the STL peak at $495 \mathrm{~Hz}$ occurs inside the resonance-based stop band, near $f_{\text {res }}$. The STL peak is followed by an introduced lowfrequency coincidence dip at $542 \mathrm{~Hz}$ at the end of the stop band, where the repeated bending wave mode cuts on for $\Gamma$ in Fig. 2a, as discussed in [25]. For $f_{\text {res }}=1000 \mathrm{~Hz}$, the STL peak occurs at $960 \mathrm{~Hz}$ and lies inside the transition stop band. The STL dip is not predicted at the end of this stop 


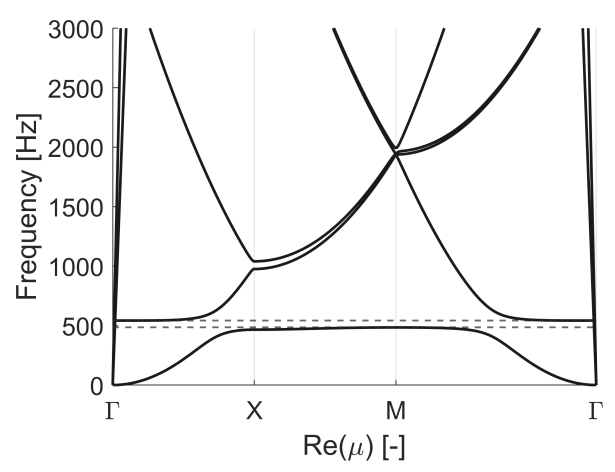

(a) $f_{\text {res }}=500 \mathrm{~Hz}$

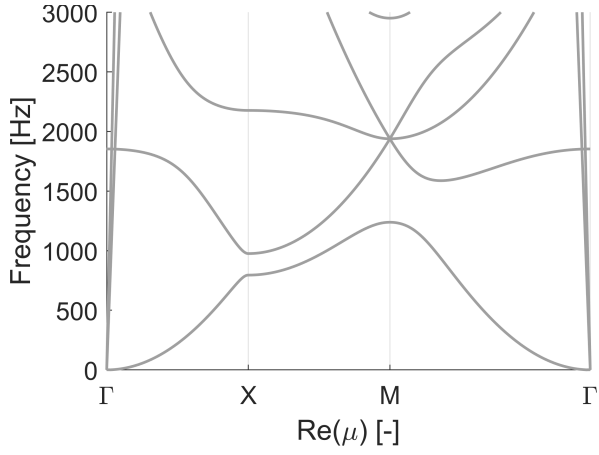

(d) $f_{\text {res }}=2000 \mathrm{~Hz}$

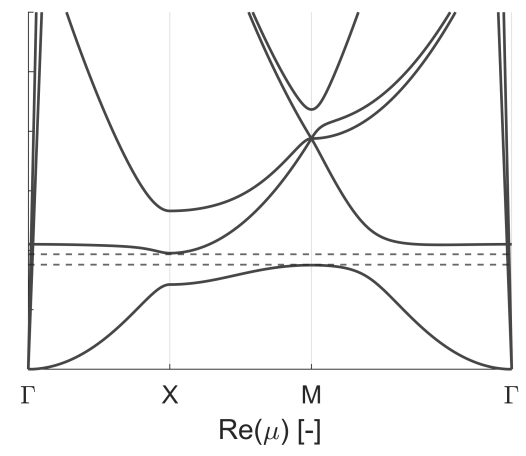

(b) $f_{\text {res }}=1000 \mathrm{~Hz}$

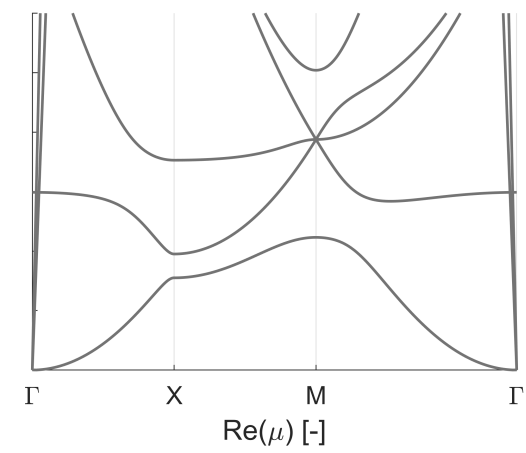

(c) $f_{\text {res }}=1500 \mathrm{~Hz}$

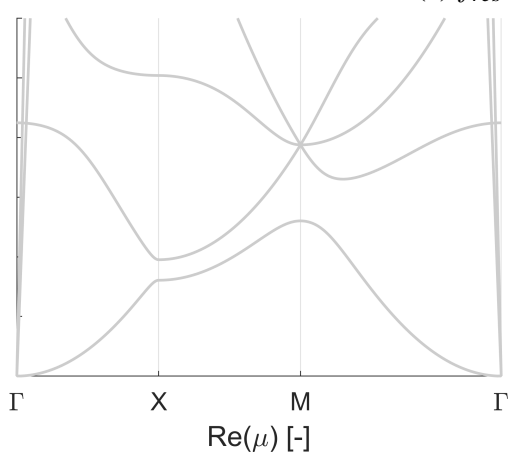

(e) $f_{\text {res }}=2500 \mathrm{~Hz}$

Fig. 2: Dispersion curves along the IBC for varying $f_{\text {res }}$. Bending wave stop bands (dashed lines) are obtained for (a) $f_{\text {res }}=500 \mathrm{~Hz}$ and (b) $f_{\text {res }}=1000 \mathrm{~Hz}$, but not for (c) $f_{\text {res }}=1500 \mathrm{~Hz}$, (d) $f_{\text {res }}=2000 \mathrm{~Hz}$ or (e) $f_{\text {res }}=2500 \mathrm{~Hz}$.

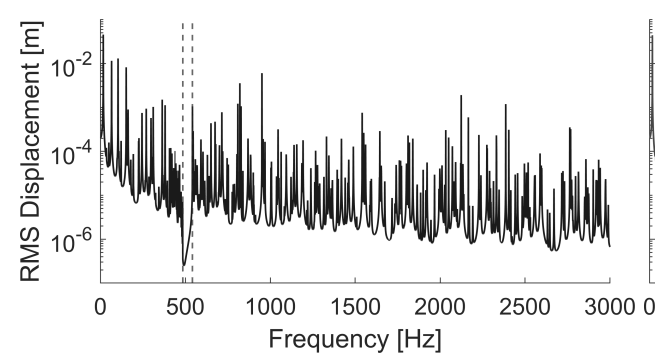

(a) $f_{\text {res }}=500 \mathrm{~Hz}$

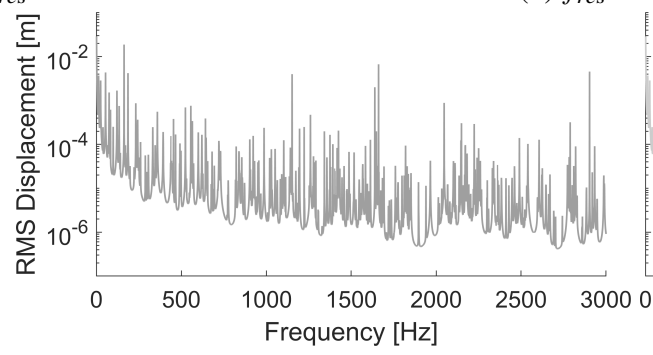

(d) $f_{\text {res }}=2000 \mathrm{~Hz}$

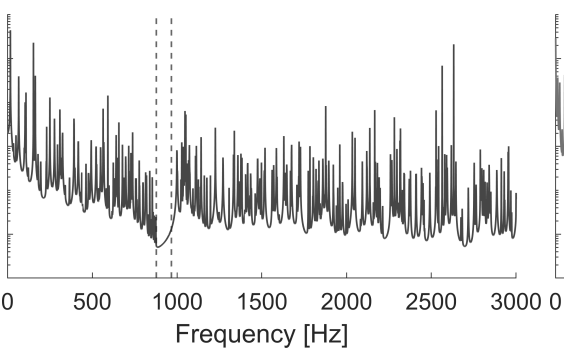

(b) $f_{\text {res }}=1000 \mathrm{~Hz}$

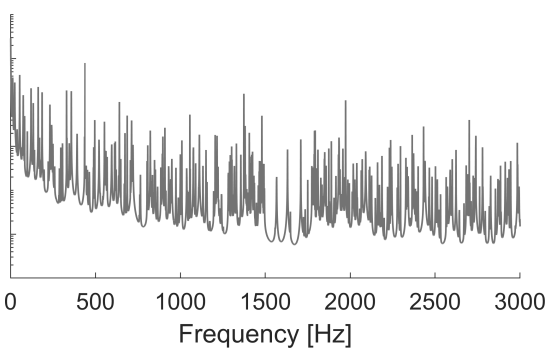

(c) $f_{\text {res }}=1500 \mathrm{~Hz}$

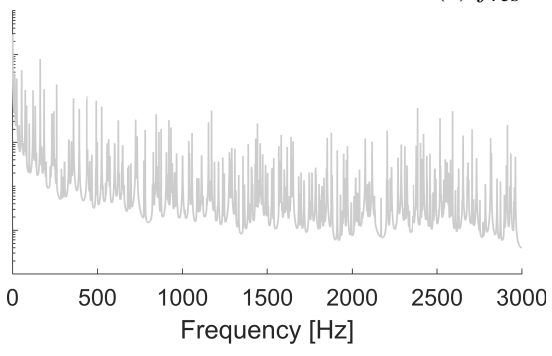

(e) $f_{\text {res }}=2500 \mathrm{~Hz}$

Fig. 3: RMS average out-of-plane plate displacement of the finite plates excited by a point force, for varying $f_{\text {res }}$. Vibration attenuation and absence of modes is obtained in the stop bands (dashed lines) for (a) $f_{\text {res }}=500 \mathrm{~Hz}$ and (b) $f_{\text {res }}=1000 \mathrm{~Hz}$.

band, but at a higher frequency of $1051 \mathrm{~Hz}$, where vibroacoustic coincidence occurs between the normally incident acoustic plane wave and the repeated bending wave solution at $\Gamma$ in Fig. 2b. For $f_{\text {res }}=1500 \mathrm{~Hz}$, the stop band has disappeared, but an STL peak is still obtained at $1368 \mathrm{~Hz}$, followed by a coincidence dip at $1496 \mathrm{~Hz}$. This STL peak for the infinite periodic plate is now predicted in absence of a stop band, in a frequency range in which bending modes are again allowed and in which local vibration suppression no longer amounts to global vibration reduction. Similar observations were made for a phononic crystal plate with periodic point-mass additions in [23] in which the STL peak emerged 

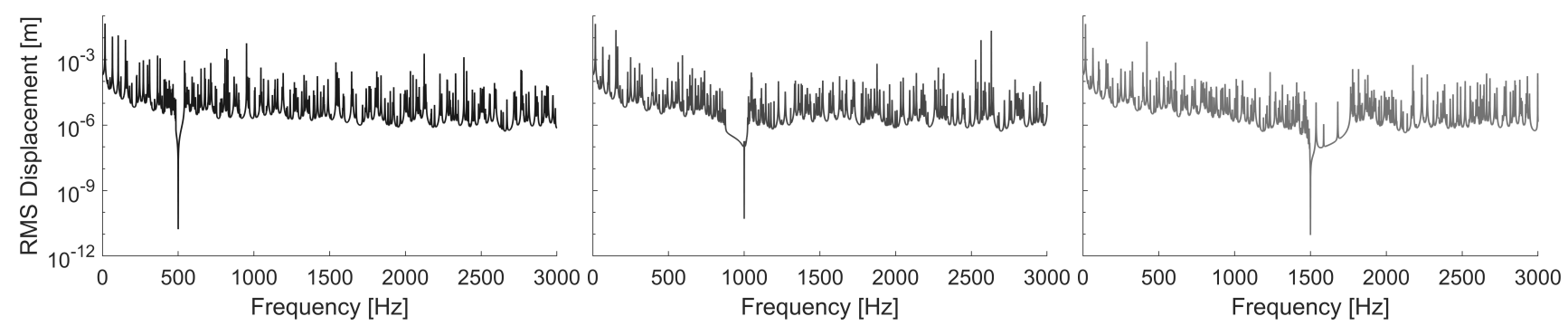

(a) $f_{\text {res }}=500 \mathrm{~Hz}$

(b) $f_{\text {res }}=1000 \mathrm{~Hz}$

(c) $f_{\text {res }}=1500 \mathrm{~Hz}$

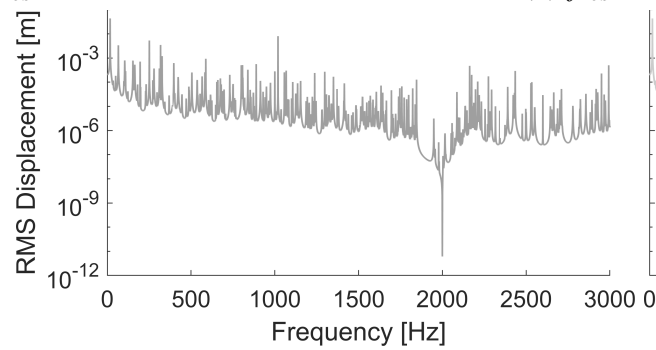

(d) $f_{\text {res }}=2000 \mathrm{~Hz}$

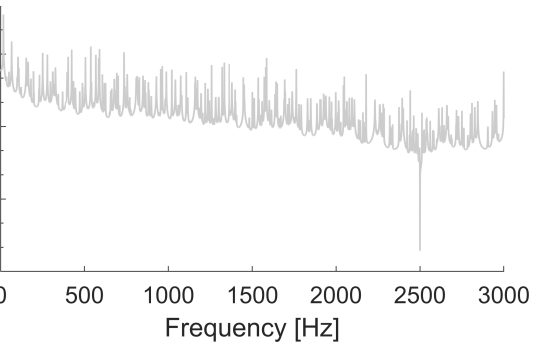

(e) $f_{\text {res }}=2500 \mathrm{~Hz}$

Fig. 4: RMS average out-of-plane displacement of the resonator bases of the finite plates excited by a point force, for varying $f_{\text {res }}$, indicating local vibration suppression at $f_{\text {res }}$ for all resonator tuning frequencies.

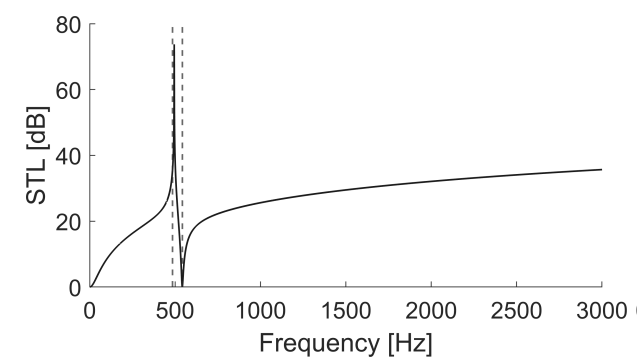

(a) $f_{\text {res }}=500 \mathrm{~Hz}$

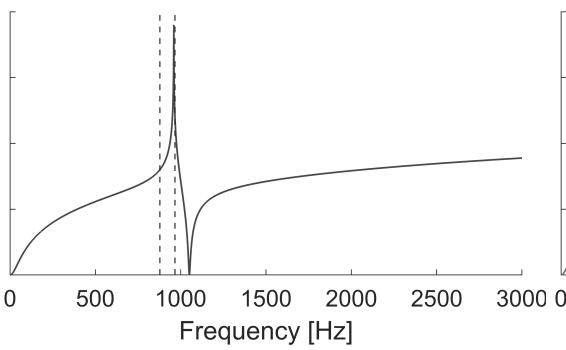

(b) $f_{\text {res }}=1000 \mathrm{~Hz}$

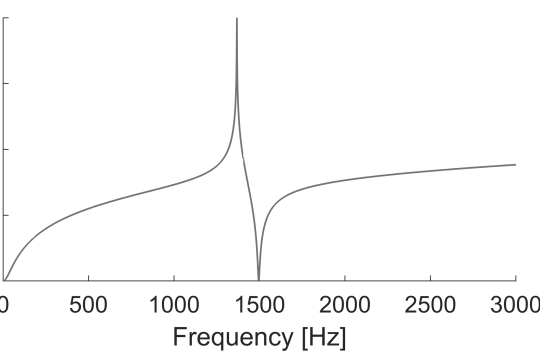

(c) $f_{\text {res }}=1500 \mathrm{~Hz}$

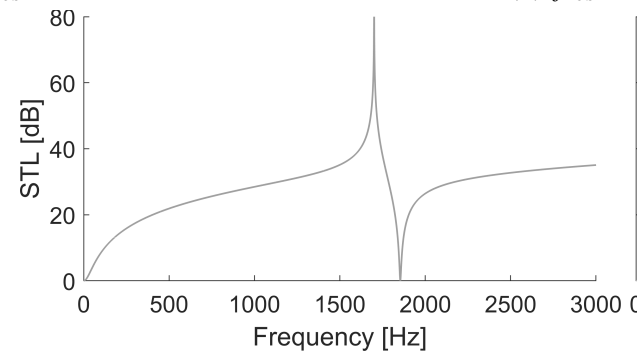

(d) $f_{\text {res }}=2000 \mathrm{~Hz}$

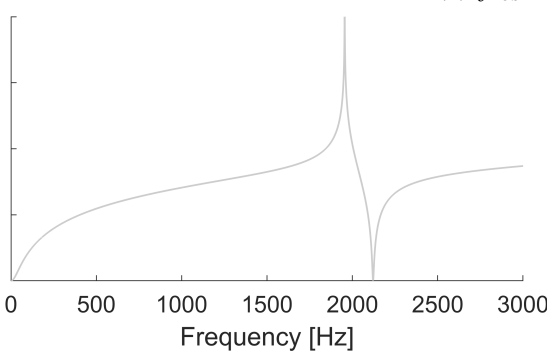

(e) $f_{\text {res }}=2500 \mathrm{~Hz}$

Fig. 5: STL of the infinite plates for varying $f_{\text {res }}$, showing a clear STL peak for all considered $f_{\text {res }}$, followed by an STL dip, regardless of the sub-wavelength criterion or presence of a bending wave stop band (dashed lines).

from an induced non-efficiently radiating sub-wavelength vibration pattern. With further increasing $f_{\text {res }}$, and thus $f_{\text {ratio }}$, the peak STL frequency is increasingly lowered relative to $f_{\text {res }}$, and the dispersion curves no longer allow predicting the frequency range of the STL peak. In line with $[13,16,21,22]$ STL enhancements can thus be predicted for infinite periodic plates with periodically added resonators, also when violating the sub-wavelength criterion. However, an increasing downshift of the predicted STL peak frequency compared to $f_{\text {res }}$ occurs with increasing $f_{\text {ratio }}$, which is in line with the observations of Xiao et al. [22].
Although the STL of the infinite periodic structures shows a clear peak regardless of the sub-wavelength criterion, for real-life applications, a finite plate of realizable dimensions needs to be considered. This allows including the resonant, structural modal response as well as finitesize effects $[24,31,32]$. The finite plate STL for a subwavelength $f_{\text {res }}=500 \mathrm{~Hz}$ corresponds well to the infinite plate STL prediction (Fig. 6), as was also found by the authors in [4]. The STL peak and dip are retrieved and no influence of bending modes is present inside the stop band. For $f_{\text {res }}=1000 \mathrm{~Hz}$, some influence of structural modes is 


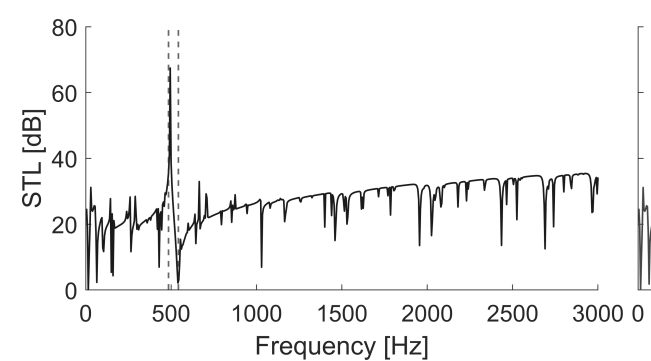

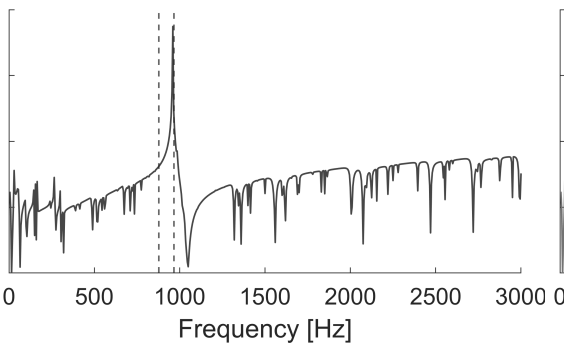

(b) $f_{\text {res }}=1000 \mathrm{~Hz}$

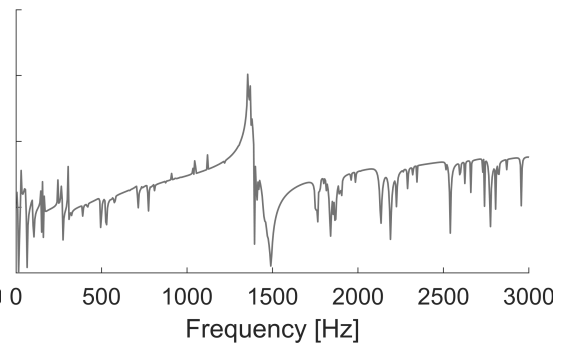

(c) $f_{\text {res }}=1500 \mathrm{~Hz}$

(a) $f_{\text {res }}=500 \mathrm{~Hz}$

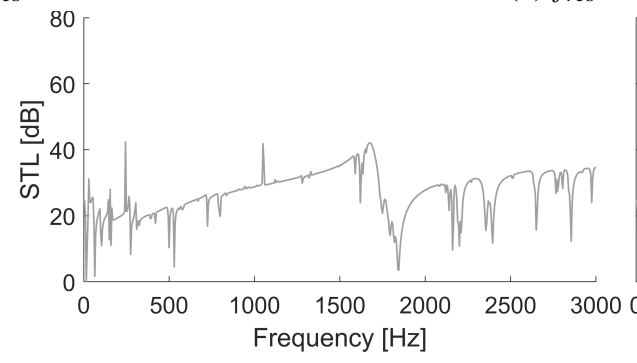

(d) $f_{\text {res }}=2000 \mathrm{~Hz}$

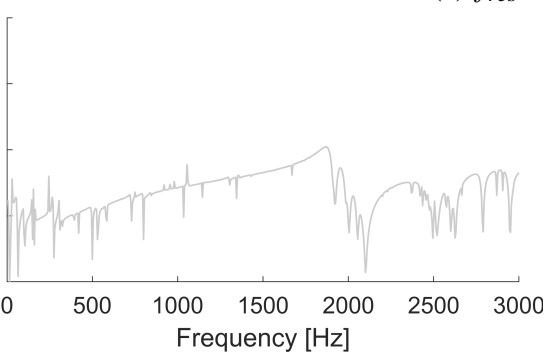

(e) $f_{\text {res }}=2500 \mathrm{~Hz}$

Fig. 6: STL of the finite plates for varying $f_{\text {res }}$, showing a vanishing STL peak when violating the sub-wavelength criterion.

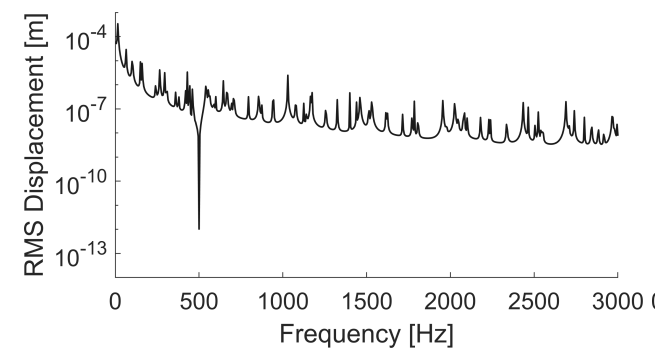

(a) $f_{\text {res }}=500 \mathrm{~Hz}$

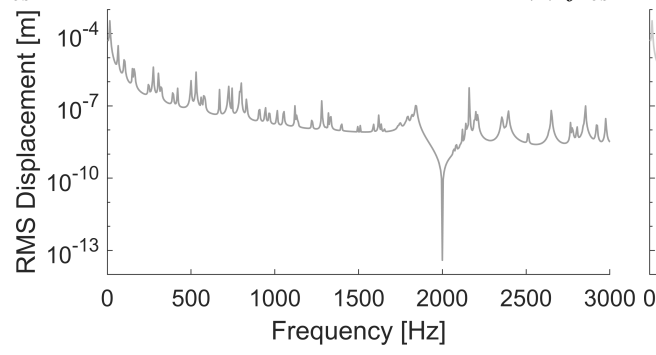

(d) $f_{\text {res }}=2000 \mathrm{~Hz}$

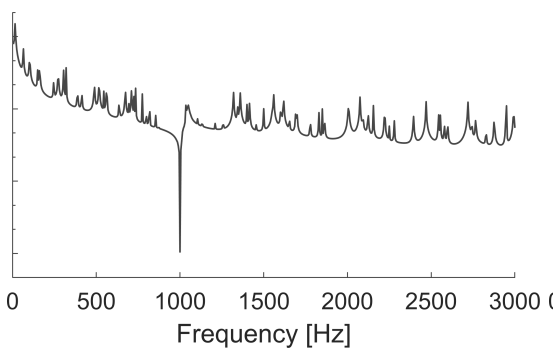

(b) $f_{\text {res }}=1000 \mathrm{~Hz}$

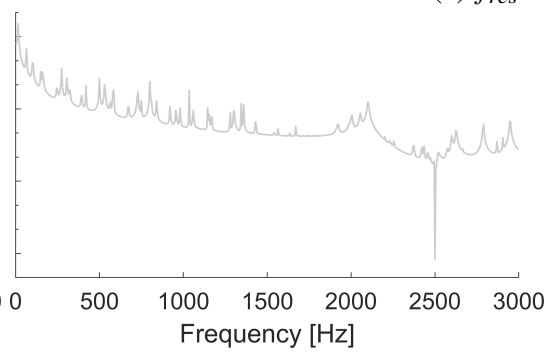

(e) $f_{\text {res }}=2500 \mathrm{~Hz}$

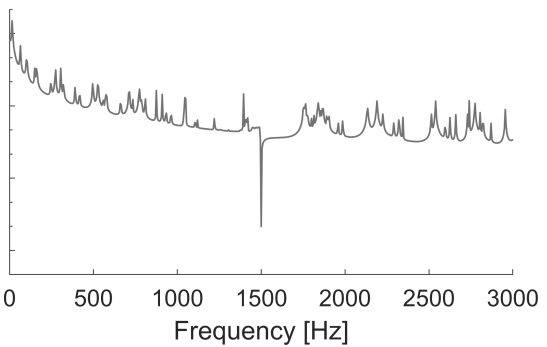

(c) $f_{\text {res }}=1500 \mathrm{~Hz}$

Fig. 7: RMS average out-of-plane displacements of the resonator bases of the finite plates excited by a normally incident acoustic plane wave, for varying $f_{\text {res }}$, indicating local vibration suppression at $f_{\text {res }}$.

present in the frequency range between STL peak and dip as free bending wave propagation is allowed after $967 \mathrm{~Hz}$. However, when the sub-wavelength criterion is further violated, for $f_{\text {res }}=1500 \mathrm{~Hz}$ and higher, the clear STL peak which was predicted for the infinite periodic structure, vanishes. Since the STL peak of the infinite periodic structure is predicted in a frequency range in which free bending wave propagation is allowed, the performance is more and more affected by the presence of bending modes of the finite partition, which lead to resonant sound transmission negatively impacting the STL peak predicted for the infinite periodic structure. Besides disappearing STL improvements, further increasing $f_{\text {res }}$ also leads to broadening zones of reduced STL after the STL peak, which is undesirable for noise reduction. Similar deviations between predicted infinite and finite plate STL performance enhancements were also found for the phononic crystal plate with periodic point mass additions in [23].

Inspecting the vibration response of the resonator bases (Fig. 7) it is clear that, although local vibration suppression is achieved at $f_{r e s}$, non-sub-wavelength spaced resonators no longer enable guaranteed stop bands, global vibration suppression or effective STL improvements around $f_{\text {res }}$. These observations underline the importance of verifying the valid- 
ity of the use of infinite periodic structure modeling when predicting the performance of and, especially, when designing metamaterial partitions for targeted noise and vibration reduction.

\section{Conclusion}

Locally resonant metamaterial partitions are often analyzed and designed using infinite periodic structure theory. While a non-sub-wavelength resonator spacing has been reported to still enable STL enhancements in some works, it was shown in this work that care should be taken in the analysis and design of locally resonant metamaterial partitions in such cases. Not only can the predicted STL peak shift considerably down in frequency with respect to the tuned resonator frequency for an increasing frequency ratio, the STL peak for non-sub-wavelength added resonators predicted using infinite periodic structure modeling is no longer guaranteed for their finite metamaterial partition counterparts. As a consequence, sub-wavelength added resonators are desirable when targeting tuned vibration as well as noise reduction with locally resonant metamaterial partitions and finite-size effects should be assessed in the case of non-sub-wavelength resonator spacing.

\section{Acknowledgements}

The Research Fund KU Leuven is gratefully acknowledged for its support. This research was partially supported by Flanders Make, the Strategic Research Center for the manufacturing industry. The research of E. Deckers is funded by a grant from the Research Foundation - Flanders $(12 \mathrm{D} 2618 \mathrm{~N})$

\section{References}

[1] Liu, Z., Zhang, X., Mao, Y., Zhu, Y. Y., Yang, Z., Chan, C. T., and Sheng, P., 2000. "Locally resonant sonic materials". Science, 289(5485), pp. 1734-1736.

[2] Hussein, M. I., Leamy, M. J., and Ruzzene, M., 2014. "Dynamics of phononic materials and structures: Historical origins, recent progress, and future outlook". Appl Mech Rev, 66(4), p. 040802.

[3] Xiao, Y., Wen, J., and Wen, X., 2012. "Sound transmission loss of metamaterial-based thin plates with multiple subwavelength arrays of attached resonators". $J$ Sound Vib, 331(25), pp. 5408-5423.

[4] Van Belle, L., Claeys, C., Deckers, E., and Desmet, W., 2019. "The impact of damping on the sound transmission loss of locally resonant metamaterial plates.”. $J$ Sound Vib, 461, p. 114909.

[5] de Melo Filho, N. G. R., Claeys, C., Deckers, E., and Desmet, W., 2019. "Realisation of a thermoformed vibro-acoustic metamaterial for increased stl in acoustic resonance driven environments". Appl Acoust, 156, pp. 78-82.

[6] Hall, A., Dodd, G., and Calius, E., 2017. “Diffuse field measurements of locally resonant partitions". In Proceedings of ACOUSTICS 2017.

[7] de Melo Filho, N. G. R., Van Belle, L., Claeys, C., Deckers, E., and Desmet, W., 2019. "Dynamic mass based sound transmission loss prediction of vibroacoustic metamaterial double panels applied to the mass-air-mass resonance". J Sound Vib, 442(3), pp. 2844.

[8] de Melo Filho, N. G. R., Claeys, C., Deckers, E., and Desmet, W., 2020. "Metamaterial foam core sandwich panel designed to attenuate the mass-spring-mass resonance sound transmission loss dip". Mech Syst Signal Process, 139, p. 106624.

[9] Claeys, C., Deckers, E., Pluymers, B., and Desmet, W., 2016. "A lightweight vibro-acoustic metamaterial demonstrator: Numerical and experimental investigation”. Mech Syst Signal Process, 70-71, pp. 853-880.

[10] Mace, B. R., and Manconi, E., 2008. "Modelling wave propagation in two-dimensional structures using finite element analysis". J Sound Vib, 318(4-5), pp. 884-902.

[11] Assouar, B., Oudich, M., and Zhou, X., 2016. "Acoustic metamaterials for sound mitigation". $C R$ Phys, 17(5), pp. 524-532.

[12] Zhang, H., Wen, J., Xiao, Y., Wang, G., and Wen, X., 2015. "Sound transmission loss of metamaterial thin plates with periodic subwavelength arrays of shunted piezoelectric patches". J Sound Vib, 343, pp. 104-120.

[13] Li, P., Yao, S., Zhou, X., Huang, G., and Hu, G., 2014. "Effective medium theory of thin-plate acoustic metamaterials". J Acoust Soc Am, 135(4), pp. 1844-1852.

[14] Wang, T., Sheng, M., and Qin, Q., 2017. "Sound transmission loss through metamaterial plate with lateral local resonators in the presence of external mean flow". $J$ Acoust Soc Am, 141(2), pp. 1161-1169.

[15] Oudich, M., Zhou, X., and Assouar, M. B., 2014. "General analytical approach for sound transmission loss analysis through a thick metamaterial plate". J Appl Phys, 116(19), p. 193509.

[16] Li, J., and Li, S., 2017. "Sound transmission through metamaterial-based double-panel structures with poroelastic cores". Acta Acust United Acust, 103(5), pp. 869-884.

[17] Vazquez Torre, J. H., Brunskog, J., and Cutanda Henriquez, V., 2020. "An analytical model for broadband sound transmission loss of a finite single leaf wall using a metamaterial". J Acoust Soc Am, 147(3), pp. 16971708 .

[18] Wester, E. C., Brémaud, X., and Smith, B., 2009. "Meta-material sound insulation". Building Acoustics, 16(1), pp. 21-30.

[19] Xiao, Y., Wen, J., Wang, G., and Wen, X., 2013. “Theoretical and experimental study of locally resonant and Bragg band gaps in flexural beams carrying periodic arrays of beam-like resonators". J Vib Acoust, 135(4), p. 041006.

[20] Claeys, C., Vergote, K., Sas, P., and Desmet, W., 2013. "On the potential of tuned resonators to obtain lowfrequency vibrational stop bands in periodic panels". 
J Sound Vib, 332(6), pp. 1418-1436.

[21] Liao, Y., Chen, Y., Huang, G., and Zhou, X., 2018. "Broadband low-frequency sound isolation by lightweight adaptive metamaterials". J Appl Phys, 123(9), p. 091705.

[22] Xiao, Y., Guo, J., and Wen, J., 2019. "Sound transmission loss of metamaterial plates with periodically attached local resonators". In Proceedings of INTERNOISE, Vol. 259, pp. 1018-1026.

[23] Van Belle, L., Claeys, C., Deckers, E., and Desmet, W., 2019. "The acoustic insulation performance of infinite and finite locally resonant metamaterial and phononic crystal plates". In MATEC Web of Conferences, Vol. 283, EDP Sciences.

[24] Fahy, F. J., 2012. Sound and structural vibration: radiation, transmission and response. Academic press.

[25] Claeys, C., Sas, P., and Desmet, W., 2014. "On the acoustic radiation efficiency of local resonance based stop band materials". J Sound Vib, 333(14), pp. 32033213.

[26] Zienkiewicz, O. C., Taylor, R. L., and Zhu, J. Z., 2005. The finite element method: its basis and fundamentals. Elsevier.

[27] Deckers, E., Jonckheere, S., Van Belle, L., Claeys, C., and Desmet, W., 2018. "Prediction of transmission, reflection and absorption coefficients of periodic structures using a hybrid wave based-finite element unit cell method". J Comput Phys, 356, pp. 282-302.

[28] De Langhe, K., Moser, C., Boeykens, R., and Kucukcoskun, K., 2016. "Sound transmission loss predictions of aircraft panels: an update on recent technology evolutions". In Proceedings of INTERNOISE, Vol. 253, pp. 3354-3365.

[29] Miccoli, G., Vansant, K., and Bertolini, C., 2014. "A validation of some recent BEM and FEM techniques for predicting exterior acoustic transfer functions for a mockup of an engine installed in the engine bay". In Proceedings of ISMA2014.

[30] Xiao, Y., Wen, J., and Wen, X., 2012. "Flexural wave band gaps in locally resonant thin plates with periodically attached springmass resonators". J Phys D Appl Phys, 45(19), p. 195401.

[31] Hopkins, C., 2012. Sound insulation. Routledge.

[32] Villot, M., Guigou, C., and Gagliardini, L., 2001. "Predicting the acoustical radiation of finite size multilayered structures by applying spatial windowing on infinite structures". J Sound Vib, 245(3), pp. 433-455.
2 Dispersion curves along the IBC for varying $f_{\text {res }}$. Bending wave stop bands (dashed lines) are obtained for (a) $f_{\text {res }}=500 \mathrm{~Hz}$ and (b) $f_{\text {res }}=1000 \mathrm{~Hz}$, but not for (c) $f_{\text {res }}=$ $1500 \mathrm{~Hz}$, (d) $f_{\text {res }}=2000 \mathrm{~Hz}$ or (e) $f_{\text {res }}=$ 2500 Hz. . . . . . . . . . . . . .

3 RMS average out-of-plane plate displacement of the finite plates excited by a point force, for varying $f_{\text {res }}$. Vibration attenuation and absence of modes is obtained in the stop bands (dashed lines) for (a) $f_{\text {res }}=500 \mathrm{~Hz}$ and (b) $f_{\text {res }}=1000 \mathrm{~Hz} \ldots \ldots \ldots \ldots$

4 RMS average out-of-plane displacement of the resonator bases of the finite plates excited by a point force, for varying $f_{\text {res }}$, indicating local vibration suppression at $f_{\text {res }}$ for all resonator tuning frequencies. . . . . . 6

5 STL of the infinite plates for varying $f_{\text {res }}$, showing a clear STL peak for all considered $f_{\text {res }}$, followed by an STL dip, regardless of the sub-wavelength criterion or presence of a bending wave stop band (dashed lines). . . .

6 STL of the finite plates for varying $f_{\text {res }}$, showing a vanishing STL peak when violating the sub-wavelength criterion. . . . . . . .

7 RMS average out-of-plane displacements of the resonator bases of the finite plates excited by a normally incident acoustic plane wave, for varying $f_{\text {res }}$, indicating local vibration suppression at $f_{r e s} \ldots \ldots \ldots \ldots$

\section{List of Tables}

1 Properties of the aluminum plate host structure. 3

2 Tuned natural frequencies $f_{\text {res }}$ of the resonators and corresponding frequency ratios

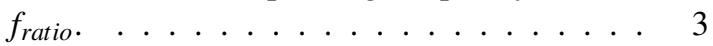

3 Tuned resonator frequencies, frequency ratios, predicted bending wave stop bands, and STL peak and dip frequencies $f_{\text {peak }}$ and $f_{\text {dip }}$ for the infinite plates with periodically added resonators. . . . . . . . . . . . 4

\section{List of Figures}

1 Plate with periodically added mass-spring resonators (a) and its representative unit cell (b) . . . . . . . . . . . 3 